\title{
Drama und Theater in der Fremd- und Zweitsprachenlehre
}

\author{
Blick zurück nach vorn
}

\section{Manfred Schewe}

\begin{abstract}
Zusammenfassung
Zwischen dem Bereich Drama/Theater und dem Bereich Fremdsprachenvermittlung gibt es seit jeher Verbindungslinien. Zumindest war der Lehrer immer schon ein Akteur, der den Schülern etwas so 'vorzuspielen' versuchte, dass die Aufmerksamkeit des Lernerpublikums gebannt blieb; und eigentlich haben Lehrer und Schüler im fremdsprachlichen Unterricht immer schon 'Theater' gespielt, indem sie so taten, als ob die Unterhaltung in der fremden Sprache für sie natürlich sei. Der folgende Beitrag zeichnet wichtige Entwicklungsetappen des Brückenbaus zwischen den Bereichen Drama/Theater und Fremd-/Zweitsprachenlehre seit Mitte des 19. Jahrhunderts nach. Es wird davon ausgegangen, dass 'Drama und Theater in der Fremd-/Zweitsprachenlehre' sich nunmehr als eines der vielen Anwendungsfelder etabliert hat, die mit dem Fach- und Sammelbegriff 'Applied Theatre' erfasst werden. Der Begriff bezieht sich auf das breite Spektrum von Individuen, Gruppen und Institutionen, für die das Theater als Kunstform nicht reiner Selbstzweck ist, sondern zentraler Bezugspunkt und Inspirationsquelle für drama-/theaterbezogene Aktivitäten. Durch solche Aktivitäten sollen im jeweiligen Anwendungsfeld ganz bestimmte Ziele erreicht werden, im Falle des fremd- und zweitsprachlichen Unterrichts z.B. sprach-, literatur- und kulturbezogene Ziele. ${ }^{1}$
\end{abstract}

\section{Historische Vorläufer einer unterrichtlichen Inszenierung von Fremdsprache: 1850-1950}

Ein früher Pionier ist der französische Fremdsprachenpädagoge François Gouin (1831-1896). Auch wenn er sich nicht explizit auf den Bereich Drama und

\footnotetext{
${ }^{1}$ Dieser Beitrag versteht sich als kompakte Bündelung und insbesondere Aktualisierung von Überlegungen, die erstmalig in meinem Buch Fremdsprache inszenieren (1993) erschienen sind. Zur Ergänzung dieses kompakten Überblicks sei auf die umfangreiche Forschungsbibliographie auf der Homepage dieser Zeitschrift verwiesen. Zum Konzept 'Applied Theatre' vgl. z.B. Ackroyd 2000; Taylor 2003; Nicholson 2005.
} 
Theater bezieht, so bereitet er mit seinem Konzept doch den Boden für das Sprachhandeln in fiktiven Kontexten. In seiner Publikation L'art d'enseigner et d'étudier les langues (1897) beschreibt er eine Sprachlehrmethode, für die das kindliche Spiel zum Modell wird. Unterricht nach dieser Methode ist in erster Linie die Ausführung von zunächst einzelnen Handlungen und dann komplexeren sprachbegleiteten 'Handlungsreihen'. Indem Gouin explizit den Zusammenhang zwischen körperlicher Handlung und Sprachproduktion betont, bereitet er den Weg für die 'Handlungsorientierung' von Fremdsprachenunterricht. Titone (1968: 36) betont das Innovative an Gouin's Konzept und sieht eine deutliche Verbindung zum Bereich Drama/Theater (Hervorhebung M.S.):

The new element that Gouin brought into the teaching of modern languages was intense activity through dramatization of the sentences to be drilled. Language was no longer considered a construct of isolated pieces, something abstract to be anatomised and then pieced together again. 'Language is behavior', Gouin could say today. Therefore, association, mimicry, memorization constituted the pivotal activities of language learning.

Gouins Sprachlehrkonzept folgt allerdings einem starren Ablaufschema, lässt keine Abweichung von diesem Schema zu und somit wenig 'Spielraum' im Sinne kreativer Unterrichtsgestaltung.

Mit Blick auf den deutschen Kontext ist beachtenswert, dass bereits Mitte des 19. Jahrhunderts Ignaz Lehmann das Prinzip der 'Anschauung' in den fachdidaktischen Blickpunkt rückte und auf diese Weise, wenn auch nicht sehr explizit, eine Verbindung zum Bereich Drama/Theater schuf (Hervorhebungen M.S.):

Durch die Anschauungsmethode werden die gewünschten Lernvorgänge begünstigt; es gibt eine direkte Anschauung und eine Anschauung im Bilde; Gegenstand der Anschauung sind die nähere Umgebung des Schülers und die Dinge des Lebens; Anschauung sowie die ihr zugeordnete Form der Mimik und Gestik erleichtern das Benennen und Beschreiben in der Fremdsprache; der Schüler hat die Möglichkeit zu fremdsprachlichem Handeln. (zitiert in Schilder 1985: 56)

Im bildungspolitisch-reformerischen Klima der Jahrhundertwende ist es vor allem Max Walter, der engagiert für die Verknüpfung von Handlung und Anschauung mit dem sprachlichen Ausdruck plädiert. Er hält große Stücke auf das methodische Vorgehen von Gouin, warnt aber vor dem ausschließlichen Einsatz seiner 'geschlossenen' Methode:

Die 'Gouinschen Reihen' sind sehr wertvoll für den Unterricht, dürfen diesem aber nicht ausschließlich zugrunde gelegt werden, wie dies Gouin wünschte, da es zu einförmig wäre, die ganze Spracherlernung in Reihenbildungen aufzulösen. Im Unterricht ist vielmehr eine gesunde Abwechslung nötig, da sie die Schüler belebt und erfrischt und sie vor Ermüdung bewahrt. (Walter 1931, zitiert in Schilder 1985: 72) 
Das Gouinsche Konzept wird von Walter erweitert. Anstatt Handlungen repetitiv nacheinander auszuführen, werden diese unterbrochen durch dialogische Szenen, bei deren Vorbereitung und Vorführung die Schüler ihrer Einbildungskraft freien Lauf lassen können. Ein Kunstbild wird z.B. zum Anlass für eine Inszenierung; die 'Rollen' der auf dem Bild sichtbaren Figuren werden von den Schülern übernommen und die abgebildeten Tätigkeiten bzw. die dazu passenden (erfundenen) Gesprächshandlungen von ihnen ausgeführt. Für Walter sind weitherhin die Dramatisierung von Lesestoffen und die Aufführung von Theaterstücken gute Möglichkeiten, um eine Vertiefung von Anschauung und Handlung und damit eine Haftung von sprachlichen Ausdrücken zu bewirken. Für ihn steht fest, dass Schüler ihre Phantasie ausagieren können sollten und überhaupt die Vorstellungskraft als Mittel zur Förderung des Unterrichts weit mehr herangezogen werden sollte. Zwar weiß sich Walter intuitiv auf einer richtigen didaktischen Spur, doch die Legitimation von Inszenierungen als festen Bestandteil des Fremdsprachenunterrichts bereitet ihm noch Probleme. Er fürchtet, dass das 'freie Spiel' den Rahmen schulischer Ordnung leicht zu sprengen droht.

In Ernst Ottos (1921) Methodik und Didaktik des neusprachlichen Unterrichts steht ein Begriff im Vordergrund, der auf die Möglichkeit vorausdeutet, einen hohen Grad an Einfühlung in eine fremde Kultur, Literatur und Sprache zu erreichen, indem in fiktiven (fremden) Kontexten Handlungserfahrungen gemacht werden:

Es ist dies der Begriff der 'sprachlichen Einfühlung', mit der eine Erschließung 'fremden Seelenlebens' erreicht werden soll: 'Die Einfühlung in und durch die Sprache geschieht auf das Vollkommenste, wenn sie durch die Handlung unterstützt wird.' Indem der Schüler Ausdrucksmittel, die über bloße Vokabeln weit hinausgehen, verwendet, wird 'die Beziehungsbedeutung der syntaktischen Beziehungsmittel beseelt und belebt.' (zitiert nach Schilder 1985: 62)

Gegen Ende der 20er Jahre scheint sich unter Neuphilologen die Auffassung herauszubilden, dass drama-/theaterbezogene Aktivitäten eine große Bereicherung für den neusprachlichen Unterricht bedeuten können. Stellvertretend seien die drei folgenden genannt:

Walter Domann (1927) betrachtet 'One Act Plays' als "ein ganz neuartiges Hilfsmittel für unseren englischen Unterricht." Er zieht diese einer Ganzschrift vor, weil darin Alltagssprache verwendet wird und Ereignisse und Zustände in den Einaktern thematisiert werden, die dem zeitgenössischen Leser eher vertraut sind. Wie man sich allerdings den methodischen Einsatz dieses Hilfsmittels vorzustellen hat, bleibt vage. Leuchten auch Domanns Gründe für die Auswahl von kurzen dramatischen Texten/Einaktern durchaus ein, so bleibt er doch konkrete Inszenierungsideen für eine skriptbezogene Unterrichtsarbeit schuldig. Klassisch-dramatischen Unterrichtsstoff durch modernen zu ersetzen, bedeutet noch keine methodische Innovation.

Georg Albrecht (1929) hat beträchtliche Einwände gegen das skript- und aufführungsbezogene Arbeiten und plädiert mit Leidenschaft für den Einsatz 
des 'Stegreifspiels'. Den gesamten Fremdsprachenunterricht für Anfänger begreift Albrecht als Stegreifspiel, indem spontan auf Gegenstände gezeigt wird und Verben handelnd dargestellt werden. In fortgeschritteneren Klassen liefern Vorlagen (Lehrbuchtexte, Märchen, Geschichten) die Grundideen für ein Stegreifspiel, das nach Albrecht einen außergewöhnlichen Enthusisasmus bei den Schülern entfacht. Ziel der Unterrichtsarbeit ist nicht die textgetreue Darstellung, sondern die Schaffung eines textbezogenen Handlungsrahmens, innerhalb dessen mit verteilten Rollen improvisiert wird.

Wie Albrecht plädiert auch Karl Mathes (1929) überzeugt für den Einsatz von Stegreifspielen im Fremdsprachenunterricht. Für ihn ist es "eines der besten Mittel, die Sprechfreude zu wecken und die Sprechfreudigkeit zu fördern" (ibid. 33). Mathes geht davon aus, "daß das darstellende Spiel im Kindesalter als selbstverständliche Lebensäußerung gewertet werden muß und daß der Trieb zum nachahmenden Darstellen von der frühesten Jugend bis in die Zeit des Erwachsenseins nicht erlischt. Es steht aber auch fest, daß nach Schuleintritt das Darstellungsspiel des Kindes im allgemeinen verschwindet ..." (ibid.), womit eine 'Apperzeptionsstütze', mit anderen Worten die Möglichkeit einer tieferen Verankerung des Gelernten, ungenutzt bleibt. Zwar führt für Mathes der Weg vom 'freien, stegreifartig gestalteten Spiel' organisch zur Darstellung eines Stückes mit vorgeschriebenen Rollen, aber das Auswendiglernen von Rollen soll erst in der Oberstufe geschehen. Und dieses betrachtet er als "kein wirkliches Erarbeiten und wird immer im Gegensatz stehen zu dem natürlichen, kindertümlichen, von dem Schülerdarsteller selbst geschaffenen Ausdruck des Stegreifspiels" (ibid. 34) ... "Der im Stegreif auftretende Schüler soll von innen heraus, aus dem Erfassen des Zusammenhangs heraus sprechen. Das Wort, das er formt, ist die Hauptsache. Angelerntes 'Schauspielern' darf nicht vorkommen" (ibid. 36). Mathes' Überlegungen kreisen somit um das Thema 'Prozess- und Produktorientierung', das im Bereich der Drama- und Theaterpädagogik bis heute immer wieder Anlass zu kontroversen Diskussionen gegeben hat.

Während des Dritten Reiches geraten reformpädagogisch-handlungsorientierte Ansätze in den Hintergrund. In den drei Jahrzehnten nach dem 2. Weltkrieg scheint eine spielorientierte Unterrichtsgestaltung kaum diskutiert zu werden, wie sich exemplarisch anhand der Zeitschrift Praxis des neusprachlichen Unterrichts zeigen lässt:

In den 25 Jahrgängen, die bis 1978 erschienen sind und in dem auf mehr als 5000 Seiten fachdidaktische Themen behandelt werden, finden sich nicht mehr als drei kleinere Beiträge ... oder rund zwanzig Seiten, in denen vom Spiel bzw. vom darstellenden Spiel als Bestandteil und Mittel des Fremdsprachenunterrichts die Rede ist. (Hoppe 1984: 289)

Offenere Unterrichtsformen im Sinne einer Inszenierung von Fremd- und/oder Zweitsprache geraten erst wieder Ende der 70er Jahre verstärkt in die fachdidaktische Aufmerksamkeit - zu einer Zeit, in der Kontextualismus und Pragmalinguistik eine Erweiterung der linguistischen Grundlagen bewirken, die funktionelle Bedeutung sprachlicher Formen/Strukturen in den Vordergrund 
gerät und das sprachliche System als Instrument und Rahmen menschlichen Handelns (Stichworte: Kontext, Situation, Sprecherrollen, außersprachliche und parasprachliche Mittel, Textsorten etc.) gesehen wird.

\section{Entwicklungen seit Ende der 70er Jahre}

Ende der 70er / Anfang der 80er Jahre erscheinen im Zuge der kommunikativen Wende in Deutschland Publikationen, die sich mit verschiedenen Spieltypen auseinandersetzen, zum Beispiel mit dem 'Darstellenden Spiel' (Macht u.a. 1977), 'Lern- und Rollenspiel' (Löffler 1979; Spier 1981), 'Handlungsspiel' (Lohfert 1983). Wenn von einer expliziten Anbindung an den Bereich Drama/Theater dabei kaum die Rede sein kann, so mehren sich die Anzeichen dafür allerdings im Laufe der 80er Jahre. Das belegen zum Beispiel die Themenschwerpunkte "Fremdsprachliches Theater in der Schule" in der Zeitschrift Der Fremdsprachliche Unterricht (86/1987) und "Theater im Unterricht Deutsch als Fremdsprache" in der Zeitschrift Informationen Deutsch als Fremdsprache (4/1988). In dieser Ausgabe von "info daf" wird erstmalig der Begriff 'dramapädagogisch' in die Fachdiskussion eingebracht und somit die Theorie und Praxis von 'Drama-in-Education' zum neu entdeckten Bezugsfeld für die Fremdsprachendidaktik.

Der Grund für das verstärkte fremdsprachendidaktische Interesse am Bereich Drama/Theater ist sicherlich gekoppelt an die Wirkung von Publikationen aus dem englischsprachigen Sprachraum (vgl. beispielsweise Maley/Duff 1978; Karbowska Hayes 1984; McRae 1985; Dougill 1987; Butterfield 1989), erklärt sich aber auch, so Esselborn (1988), auf dem Hintergrund der in diesen Jahren intensiv diskutierten 'alternativen Sprachlehr-/lernmethoden' (z.B. Total Physical Response (Asher 1986), Suggestopädie, Community Language Learning), die bewusst Aspekte wie Körperlichkeit, Gefühl, Gestik und Mimik integrieren. Eine kritisch-vergleichende Übersicht dieser Methoden findet sich beispielsweise bei Ortner (1998).

Während es sich bei den Veröffentlichungen der 70er und 80er Jahre vornehmlich um Spielesammlungen und unterrichtspraktische Ratgeberliteratur handelt, findet seit Anfang der 90er-Jahre eine intensivere wissenschaftliche Auseinandersetzung mit der Wechselbeziehung zwischen den Bereichen Drama/Theater und Fremd-/ Zweitsprachenunterricht statt. Die Veröffentlichung Fremdsprache inszenieren (Schewe 1993), eine gründliche Auseinandersetzung mit Geschichte, Theorie und Praxis von 'Drama-in-Education', trägt den Untertitel 'Zur theoretischen Fundierung einer dramapädagogischen Lehr- und Lernpraxis.' Die Untermauerung dieser Lehr- und Lernpraxis erfolgt anhand von Erkenntnissen aus der Fremd- und Zweitsprachendidaktik und verschiedenen Bezugsdisziplinen, z.B. Lernpsychologie, Neuropsychologie, Sozial- und Individualpsychologie, Psycholinguistik, Psychophysiologie. Zeitgleich erscheint der Sammelband Towards Drama as a Method in the Foreign Language Classroom (hrsg. von Schewe/Shaw 1993), zu dem Autoren aus sieben verschiedenen Ländern beitragen, um das komplexe Feld drama-/ theaterbezogener 
Unterrichtsaktivitäten weiter abzustecken und zu systematisieren.

Die folgenden Jahre bis heute sind geprägt von weiterer Konzeptualisierung und Systematisierung, was den Brückenbau zwischen den Bereichen Drama/Theater und Fremd-/Zweitsprachenunterricht angeht. Das soll an einigen ausgewählten Beispielen veranschaulicht werden:

Im Fach Romanistik entwickelt Bernard Dufeu in Teaching Myself (1994) das Konzept einer 'Linguistischen Psychodramaturgie', das auf dem Fundament einer 'Pädagogik des Seins' (Dufeu 1982) aufbaut. In der Bezeichnung sind die beiden Bezugsfelder enthalten, auf denen dieses Konzept theoretisch fußt: Psychodrama (z.B. Moreno 1973) und Dramaturgie (z.B. Souriau 1950; Boal 1983). Die Anwendung von Erkenntnissen aus diesen Feldern im pädagogischen Kontext des Sprachlernens schränkt Dufeu allerdings wie folgt ein:

We do not provide therapy or theatre, but we use foundations and techniques which stem from these two areas, just as actors or sports people refer to other disciplines, such as relaxation and visualization, to improve the quality of their performance. (Dufeu 1994: 22f)

Im Praxisteil seines Buches beschreibt Dufeu eine Fülle von anregenden Übungen, u.a. Übungen, mit denen eine Entspannung der Lerner, Entwicklung ihres Rhythmus-Gefühls und Erweiterung ihres sprachlichen Ausdrucksvermögens bewirkt wird. Er schließt ab mit Überlegungen zum Einsatz dieses Konzepts in der Lehrerausbildung, einschließlich einer Auflistung von Schwerpunkten eines Trainings für 'LPD-Animatoren.'

Dufeus fremdpädagogisches Konzept zielt insbesondere auf die Entwicklung der Lernerpersönlichkeit ab. Lerner werden dazu ermutigt, ungewohnte Lernwege zu gehen (zum Beispiel, als Teil einer Aufwärm- und Entspannungsübung, sich auf den Boden zu legen und auf eine Phantasiereise zu gehen oder während einer Übung in einer späteren Unterrichtssequenz Halb- oder Vollmasken zu tragen). Durch die Erfahrungen, die sie dabei machen, werden sie sich z.B. ihrer kreativen (Sprach-) Handlungsfähigkeit bewusst. Dufeus Konzept ist inhaltlich verwandt mit den Lehr- und Forschungsaktivitäten seines Romanistik-Kollegen Daniel Feldhendler, der sich ebenfalls auf psychodramatische Grundlagen beruft, Aspekte des inter- und transkulturellen Lernens ins Zentrum stellt und sich dabei an einer spezifischen Theaterform orientiert: dem Playback-Theater (z.B. Feldhendler 1993; 2004).

Während Schewe/Wilms (1995) in Texte lesen, verstehen und inszenieren ${ }^{2}$ anhand der didaktischen Bearbeitung eines Romans demonstrieren, wie dramapädagogische Arbeitsweisen auch den Literaturunterricht bereichern können, reflektiert Angelika Mairose-Parovsky (1997) über den Brückenbau zwischen Drama/Theater und Kulturvermittlung. Sie legt mit ihrem Buch Transkulturelles Sprechhandeln ein Konzept für den Deutsch als FremdspracheUnterricht vor, in dem sie sich abgrenzt von den üblichen 'Rollenspielen' und für

\footnotetext{
${ }^{2}$ Ulrike Brisson setzt sich in ihrem Artikel Sansibar oder der wirkliche Grund in dieser Ausgabe von SCENARIO kritisch mit der Veröffentlichung von Schewe/Wilms auseinander.
} 
eine Unterrichtsgestaltung nach sozial- und theaterpädagogischen Prinzipien plädiert, die auf die Befähigung zu transkulturellem Sprechhandeln zielt:

Transkulturelles Sprechhandeln bedeutet [...]: Im Gewahrsam der kulturell unterschiedlichen Gestaltung der Begegnung mit dem anderen, sowie der - manchmal auch - Tarn- und Verschleierungsfunktion von diversen Routinen und Ritualen, sollen die allgemeinmenschlichen Grundzüge von Interaktionen transparent werden. Eine derartige transkulturelle Wahrnehmungsschulung hätte als Idealvorstellung das Ziel, sich selbst innerhalb des soziokulturellen Rahmens besser kennenzulernen und dadurch Kommunikationsstörungen zwischen den Kulturen vermeiden zu helfen. (1977: 80)

Die Autorin beruft sich auf diverse theoretische Bezugsquellen aus der Theaterund Sozialpädagogik, u.a. das 'Playback-Theater' nach Jonathan Fox, das 'Psychodrama' nach Jacob Levy Moreno, die 'Themenzentrierte Interaktion' nach Ruth Cohn, Augusto Boals 'Theater der Unterdrückten' und darüber hinaus auf weiter oben bereits genannte Quellen (Dufeu, Feldhendler, Schewe).

Mit den Publikationen von Kao Shin Mei/Cecily O'Neill (1998) und Elektra Tselikas (1999) wird die britische Dramapädagogik als fremd- und zweitsprachendidaktisches Bezugsfeld wieder explizit in den Blickpunkt gerückt. Kao/O'Neill benutzen den Oberbegriff 'Process Drama', um zu beschreiben, wie sich Fremd- und Zweitsprachenunterricht als szenische Improvisation gestalten lässt. Ähnlich finden sich bei Tselikas, die insbesondere vom Bezugsfeld Dramatherapie (z.B. Jennings 1998) inspiriert wird und ihr Konzept auch auf den muttersprachlichen Deutschunterricht ausweitet, zahlreiche Tipps für eine dramapädagogische Strukturierung von Unterricht.

Während dramapädagogische Konzepte in manchen Veröffentlichungen (z.B. Ortner 1998) den 'alternativen Methoden' zugeordnet werden, wird ihnen in dem Sammelband Pädagogische Konzepte für einen ganzheitlichen DaF-Unterricht (Schlemminger/Brysch/Schewe 2000) ein klarer Platz als eigenständige Konzepte innerhalb des sprachdidaktischen Fachdiskurses eingeräumt. Ein weiterer Sammelband (hrsg. von Bräuer 2002) mit dem Titel Body and Language betont die körperbezogene und - wie bereits der Sammelband von Byram/Fleming (1998) - besonders die interkulturelle Dimension drama- und theaterpädagogischen Fremdsprachenlernens. Dieses wird unter dem Gesichtspunkt der handelnden Auseinandersetzung mit unterschiedlichen Bedeutungen, Denkrichtungen und kulturellen Ausdrucksformen diskutiert. Im ersten Teil des Bandes geht es um theoretische Grundlagen eines drama/theaterbezogenen Fremdsprachenunterrichts, im zweiten um Praxismodelle auf Schul- und Hochschulebene, im dritten um interdisziplinär angelegte Theaterprojekte in universitären Kontexten.

Im Jahr 2003 erscheinen zwei Veröffentlichungen, in denen sich die Autorinnen (Even; Huber) um eine solide theoretische Fundierung drama/theaterpädagogischer Unterrichtsgestaltung bemühen und diese anhand von konkreten Beispielen aus ihrer Unterrichtspraxis an Hochschulen in Portugal, Großbritannien und den USA überzeugend veranschaulichen. Susanne Even 
geht in Drama Grammatik von einer zentralen These aus: "Dramapädagogischer Unterricht kann eine wirksame Verarbeitung fremdsprachlicher grammatischer Phänomene gewährleisten" (Even 2003: 21). Damit wirft sie neues Licht auf einen besonderen Teilbereich des Fremdsprachenunterrichts. Es geht ihr um eine Effektivierung des Grammatikunterrichts, speziell um die Aufhebung der Diskrepanz zwischen Regelwissen und Sprachkönnen. Sprachlehrer bekommen in dieser Studie, in der die Autorin sich intensiv mit der Fachdiskussion in der Sprachlehr-/lernforschung auseinandersetzt, eine Fülle von Anregungen dafür, wie dramapädagogische Mittel gezielt eingesetzt werden können, um Grammatik für Lernende lebendig und sinnhaft werden zu lassen.

$\mathrm{Zu}$ Beginn von Ruth Hubers Im Haus der Sprache wohnen. Wahrnehmung und Theater im Fremdsprachenunterricht (2003) heißt es: "Lernen ist ein ästhetischer Prozess. Etwas weniger apodiktisch formuliert: Es kommt dem Lernen zugute, wenn es als ästhetischer Prozess aufgefasst und in der Unterrichtskonzeption entsprechend organisiert wird" (ibid. 1). Hubers Studie deckt neben Linguistik und Didaktik ein breites Spektrum von Bezugswissenschaften ab: Literatur-, Musik- und Theaterwissenschaft, Neurowissenschaften, Intelligenzund Kreativitätsforschung, Soziologie, Anthropologie und Sozialpsychologie. Kapitelüberschriften wie 'Der Mensch ist ein Augentier' und 'Gehörsensibilisierung' spiegeln Hubers Anliegen: Es geht ihr um Wahrnehmungsschulung, um die Aktivierung aller Lerner-Sinne und sie demonstriert am Ende des Buches ihre Vorstellung eines "aisthetisch-ästhetisch orientierten Fremdsprachenunterrichts" an Praxismodellen, die im Laufe ihrer Arbeit mit fortgeschrittenen Deutschlernern entstanden sind.

Die Ausgabe 1/2004 der Internet-Fachzeitschrift German as a Foreign Language (www.gfl-journal.com) bündelt acht Artikel und drei Rezensionen zum Thema 'Dramapädagogik und fremdsprachlicher Deutschunterricht' und enthält eine aktualisierte Forschungsbibliographie.

\section{Zukunftsperspektiven}

Anhand des Überblicks ist deutlich geworden, dass der Brückenbau zwischen Drama/Theater und Fremd-/Zweitsprachenunterricht seit der Mitte des 19. Jahrhunderts ein entscheidendes Stück voran gekommen ist. Die kommunikative Wende gegen Ende der 70er / Anfang der 80er Jahre bringt ein verstärktes Interesse für den Bereich Drama/Theater mit sich und seit Anfang der 90er Jahre lassen sich deutlich Tendenzen zur Entwicklung einer eigenständigen drama-/theaterbezogenen Fremd- und Zweitsprachendidaktik erkennen. Das spiegelt sich in der Fülle und Qualität von einschlägigen Publikationen, aber auch in der Organisation von Fachkonferenzen (2003: Drama and Theatre in the Teaching and Learning of Language, Literature and Culture, University College Cork, Irland; 2005: Sektion Dramapädagogik auf der Internationalen Deutschlehrerkonferenz in Graz; 2007: Panel zur Drama- und Theaterpädagogik auf der ACTFL-Konferenz in Baltimore, Maryland). Aus internationaler Perspektive gesehen lässt sich feststellen, dass wesentliche Impulse für eine 
drama-/theaterbezogene Didaktik zunächst vom englischsprachigen Raum ausgingen, insbesondere der allgemein-didaktischen Teildisziplin Drama-inEducation. Der Begriff 'dramapädagogisch' - als Bezeichnung für ganzheitlich orientierte Formen sprachlichen, literarischen und (inter)kulturellen Lernens, die aus der Begegnung zwischen den Bereichen Fremdsprachenvermittlung und Drama/Theater erwachsen - hat sich in der Fachdiskussion deutschsprachiger Länder inzwischen etabliert und deckt ein sehr breites Spektrum von prozess- und produktorientierter Unterrichtsgestaltung ab. ${ }^{3}$ Auch außerhalb Westeuropas lässt sich ein zunehmendes Interesse an Aspekten einer drama/theaterbezogenen Fremd- und Zweitsprachendidaktik beobachten. ${ }^{4}$ So wird in Zukunft voraussichtlich der Aspekt kulturspezifische Lehr- und Lerntradition bzw. Drama-/ Theatertradition mehr in den Blick geraten und, parallel mit der stetig zunehmenden Zahl an drama-/theaterinteressierten Fremdsprachenpädagogen, das Bedürfnis nach fachlichem Austausch stetig wachsen. Um diesem Bedürfnis entgegen zu kommen, ist unlängst diese referierte, bilinguale Internet-Fachzeitschrift entwickelt worden.

Auf der Homepage von SCENARIO (http://scenario.ucc.ie) befindet sich u.a. eine Forschungsbibliographie mit derzeit über 600 Titeln. Sie wird regelmäßig aktualisiert und dient damit künftig als eine Art Barometer für die Fachdiskussion im Bereich 'Drama und Theater in der Fremd- und Zweitsprachenlehre'.

Mit ihren Beiträgen regen die Autoren dieser ersten Ausgabe u.a. dazu an, in der Fachdiskussion folgende Themen weiter zu beleuchten:

- Dramapädagogik für verschiedene Stufen bzw. Lernniveaus im Sinne des Europäischen Referenzrahmens, insbesondere auch für Sprachanfänger;

- Dramapädagogik und autonomes Lernen;

- Dramapädagogik als aktivierende Lehr- und Lernmethode im Umgang mit (kanonisierter bzw. verfilmter) Literatur;

- Wege zur Entwicklung einer dramapädagogischen Lehrkompetenz.

Das Spektrum möglicher Forschungsthemen ist zweifellos sehr breit. Über die vertiefende Auseinandersetzung mit solchen Themen hinaus ist allerdings auch wichtig, dass in den nächsten Jahren die Forschung selbst stärker als bisher zum Gegenstand der Fachdiskussion wird und Antworten auf folgende Fragen gegeben werden:

- Welche Konzepte bzw. quantitativen und qualitativen Methoden wurden bei der Erforschung von dramapädagogischen Fremd- und Zweitsprachenunterricht eingesetzt? Mit welchem Erfolg? Welche neuen Konzepte/Methoden sollten erprobt werden?

\footnotetext{
${ }^{3}$ Den Prozess der Erarbeitung einer auf Improvisationsarbeit basierenden Aufführung vor Publikum beschreiben beispielsweise Schewe/Scott (2003); in diesem Kontext sei auf Moodys (2002) erfrischendes Plädoyer verwiesen, Prozess und Produkt nicht als Gegensätze zu konstruieren.

${ }^{4}$ Z.B. im asiatisch-pazifischen Raum Donnery (2006).
} 
Warum das Interesse an der (Unterrichts-)Forschung wach gehalten werden sollte, daran erinnert Betty Jane Wagner (1998: 244):

“... without research we do not have a lodestar to show us which way to face as we tack our way toward more effective teaching".

Ihre Publikation Educational Drama and Language Arts. What Research Shows, in der sie primär die Forschungsanstrengungen im Bereich 'Drama/Theater und muttersprachlicher Englischunterricht' bilanziert, dürfte all denen als Anregung dienen, die künftig zur weiteren Ausmessung der Forschungslandschaft im Bereich 'Drama und Theater in der Fremd- und Zweitsprachenlehre' beitragen möchten.

\section{Bibliographie}

Ackroyd, Judith (2000): Applied Theatre: Problems and Possibilities. In: Applied Theatre Researcher 1, 2000. February 26, 2007

<http://www.gu.edu.au/centre/cpci/atr/journal/article1_number1.htm>

Albrecht, Georg (1929): Stegreifspiel im englischen Unterricht. In: Zeitschrift für französischen und englischen Unterricht, 531-534

Asher, J.J. (1986): Learning another Language through Actions. The Complete Teacher's Guidebook. Los Gatos,CA: Sky Oaks Productions

Boal, Augusto (1983): Jeux pour acteurs et non-acteurs. Paris: François Maspero

Bräuer, Gerd (Hrsg.) (2002): Body and Language. Intercultural Learning through Drama. Westport, CT / London: Ablex Publishing

Butterfield, Tony (1989): Drama through Language through Drama. Banbury: Kemble Press

Byram, Mike / Fleming, Mike (Hrsg.) (1998): Language Learning in Intercultural Perspective. Approaches through Drama and Ethnograpy. Cambridge: Cambridge University Press

Domann, Walter (1927): One Act Plays of To-Day für den Schulgebrauch. In: Zeitschrift für den französischen und englischen Unterricht, 1926/27, 33-42

Donnery, Eucharia (2006): Drama in EFL: an Intercultural- and Arts-Based Pedagogy. In: Polyglossia 11, 51-61

Dougill, John (1987): Drama Activities for Language Learning. London: Basingstoke

Dufeu, Bernard (1982): Vers une pédagogie de l'être: la pédagogie relationnelle. In: Die Neueren Sprachen 81/3, 267-289

Dufeu, Bernard (1994): Teaching Myself. Oxford: Oxford University Press

Esselborn, Karl (1988): Theater und szenisches Spiel im Fremdsprachenunterricht Deutsch. In: Informationen Deutsch als Fremdsprache (info daf) 15/4, 388-406 
Even, Susanne (2003): Drama Grammatik. Dramapädagogische Ansätze für den Grammatikunterricht Deutsch als Fremdsprache. München: iudicium

Feldhendler, Daniel (1993): Enacting Life! Proposal for a Relational Dramaturgy for Teaching and Learning a Foreign Language. In: Schewe, Manfred / Shaw, Peter (eds.): Towards Drama as a Method in the Foreign Language Classroom. Frankfurt/Main et al: Peter Lang, 171-191

Feldhendler, Daniel (2004): Playback Theater. In: Koch, Gerd / Roth Sieglinde / Vaßen Florian / Wrentschur, Michael (eds.): Theaterarbeit in sozialen Feldern. Frankfurt/Main: Brandes \& Apsel, 147-152

Gouin, François (1897): L'art d'enseigner et d'étudier les langues. Paris: Fischbacher

Hoppe, Hans (1984): Spiel im Deutsch- und Fremdsprachenunterricht. Begründungen und Kriterien für die fächerspezifische Spielauswahl und -verwendung. In: Kreuzer, Karl Josef (Hrsg.): Handbuch der Spielpädagogik. $B d$. 2. Spiel im frühpädagogischen und schulischen Bereich. Düsseldorf: Schwann, 285-303

Huber, Ruth (2003): Im Haus der Sprache wohnen. Tübingen: Max Niemeyer Verlag

Jennings, Sue E. (1998): Introduction to Dramatherapy. London: Jessica Kingsley Publications

Kao, Shin-Mei / O'Neill, Cecily (1998): Learning a Second Language through Process Drama. Stamford,CT / London: Ablex Publishing

Karbowska Hayes, Suzanne (1984): Drama as a Second Language. A Practical Handbook for Language Teachers. Cambridge: National Extension College Trust Ltd.

Löffler, Renate (1979): Spiele im Englischunterricht. Vom lehrergelenkten Lernspiel zum schülerorientierten Rollenspiel. München: Urban \& Schwarzenberg

Lohfert, Walter (1983): Kommunikative Spiele für Deutsch als Fremdsprache. Spielpläne und Materialien für die Grundstufe. München: Hueber

Macht, Konrad (1977): Das darstellende Spiel im Englischunterricht. Ansbach: Prögel

Maley, Alan / Duff, Alan (1978): Drama Techniques in Language Learning. Cambridge: University Press; Übersetzung (1985): Szenisches Spiel und freies Sprechen im Fremdsprachenunterricht. München: Hueber

Mathes, Karl (1929): Das Stegreifspiel im neusprachlichen Unterricht. In: Zeitschrift für französischen und englischen Unterricht 28, 33-43

Mairose-Parovsky, Angelika (1997): Transkulturelles Sprechhandeln. Bild und Spiel in Deutsch als Fremdsprache. Frankfurt/Main et al: Peter Lang

McRae, John (1985): Using Drama in the Classroom. Oxford: Pergamon

Moody, Douglas J. (2002): Undergoing a Process and Achieving a Product: A Contradiction in Educational Drama? In: Bräuer, Gerd (Hrsg.) (2002): Body 
and Language. Intercultural Learning Through Drama. Westport, CT / London: Ablex Publishing, 135-159

Moreno, Jacob Levy (1973): The Theater of Spontaneity. New York: Beacon Nicholson, Helen (2005): Applied Drama: The Gift of Theatre. Basingstoke: Palgrave

Ortner, Brigitte (1998): Alternative Methoden im Fremdsprachenunterricht. Lerntheoretischer Hintergrund und praktische Umsetzung. Ismaning: Max Hueber Verlag

Otto, Ernst (1921): Methodik und Didaktik des neusprachlichen Unterrichts. Versuch einer wissenschaftlichen Universitätslehre. Bielefeld/Leipzig: Velhagen \& Klasing

Schewe, Manfred (1993): Fremdsprache inszenieren. Zur Fundierung einer dramapädagogischen Lehr- und Lernpraxis. Oldenburg: Didaktisches Zentrum, Universität Oldenburg

Schewe, Manfred / Shaw, Peter (1993) (Hrsg.): Towards Drama as a Method in the Foreign Language Classroom. Frankfurt/Main et al: Peter Lang

Schewe, Manfred/Wilms, Heinz (1995): Texte lesen, verstehen und inszenieren. Stuttgart: Klett (Edition Deutsch)

Schewe, Manfred / Scott, Trina (2003): Literatur verstehen und inszenieren: Foreign Language Literature Through Drama. A Research Project. In: German as a Foreign Language (GFL) 3, 58-63

Schilder, Hans (1985): Reformbestrebungen und Wendepunkte in den Grundlagen und Methoden des Fremdsprachenunterrichts / Quellen zur Methodengeschichte. In: Englisch-Amerikanische Studien 7/1, 54-59 / 68-79

Schlemminger, Gerald / Brysch, Thomas / Schewe, Manfred Lukas (Hrsg.) (2000): Pädagogische Konzepte für einen ganzheitlichen DaF-Unterricht. Berlin: Cornelsen

Souriau, E. (1950): Lex deux cent mille situations dramatiques. Paris: Flammarion Editeur

Spier, Anne (1981): Mit Spielen Deutsch lernen. Spiele und spielerische Übungsformen für den Unterricht mit ausländischen Kindern, Jugendlichen und Erwachsenen. Königstein/Ts.: Scriptor

Taylor, Philip (2003): Applied Theatre. Creating Transformative Encounters in the Community. Portsmouth, NH: Heinemann

Tselikas, Elektra I. (1999): Dramapädagogik im Sprachunterricht. Zürich: Orell Füssli

Wagner, Betty Jane (1998): Educational Drama and Language Arts. What Research Shows. Portsmouth, NH: Heinemann

Wessels, Charlyn / Kerridge, H. (1987): The Drama Option. Language Learning with a Difference. In: Modern English Teacher 15/2, 8-15

Walter, Max (1931): Zur Methodik des Neusprachlichen Unterrichts. Marburg: Elwert [Nachdruck von Kapitel 2 "Die Handlung und ihre Entwicklung" in Schilder (1985)] 